\title{
Application of digital image correlation at the microscale in fiber-reinforced composites
}

\author{
L.P. Canal ${ }^{\text {a }}$, C. González ${ }^{\text {a,b }}$, J.M. Molina-Aldareguía ${ }^{\text {b }}$ J. Segurado ${ }^{\text {a,b }}$, J. LLorca ${ }^{\text {a,b,* }}$
}

\begin{abstract}
A B S T R A C T
Digital image correlation (DIC) is applied to analyzing the deformation mechanisms under transverse compression in a fiber-reinforced composite. To this end, compression tests in a direction perpendicular to the fibers were carried out inside a scanning electron microscope and secondary electron images obtained at different magnifications during the test. Optimum DIC parameters to resolve the displacement and strain field were computed from numerical simulations of a model composite and they were applied to micrographs obtained at different magnifications $(250 \times, 2000 \times$, and $6000 \times)$. It is shown that DIC of low-magnification micrographs was able to capture the long range fluctuations in strain due to the presence of matrix-rich and fiber-rich zones, responsible for the onset of damage. At higher magnification, the strain fields obtained with DIC qualitatively reproduce the non-homogeneous deformation pattern due to the presence of stiff fibers dispersed in a compliant matrix and provide accurate results of the average composite strain. However, comparison with finite element simulations revealed that DIC was not able to accurately capture the average strain in each phase.
\end{abstract}

\section{Introduction}

Mechanical properties of engineering materials are often dictated by phenomena which take place at the micron scale, which is the size of the relevant microstructural features (grain size, fiber or inclusion diameter, etc.). This is the case, for instance, of many deformation (grain boundary sliding, matrix-reinforcement load transfer) and fracture mechanisms (intergranular cracking, fibermatrix decohesion, inclusion fracture). Our knowledge of the role played by the microstructure in the mechanical performance of materials has benefited greatly from the information provided by different kinds of microscopy (optical, scanning and transmission electron, atomic force) and particularly from the ability to carry out in situ mechanical tests inside the microscope. This latter technique allows for recording of the sequence of the deformation and fracture events, leading to a clear, although qualitative, understanding of the interaction among the relevant microstructural features on the performance.

More precise quantitative information about the deformation and fracture processes at the micron scale can be obtained through the application of optical full-field measurement methods, e.g., digital image correlation (DIC) in combination with in situ testing inside a scanning electron microscope. DIC is nowadays a mature technique, widely used in the field of experimental mechanics to obtain the full displacement field (sometimes including the outof-plane displacements) from optical images taken at different stages of deformation [3]. Its popularity among the optical methods comes from the availability of commercial packages, the low cost of high-resolution digital cameras and the general simplicity of sample preparation and optical set-up. Accordingly, DIC has been applied in recent years to revealing the deformation and fracture mechanisms of $\mathrm{Ti}$ alloys [8], dual-phase steels [5,7], and $\mathrm{Al}$ foams [1] by analyzing secondary electron images obtained during in situ testing. Displacement correlation in DIC is based on the presence of a random speckle pattern on the specimen surface whose movement is tracked and compared with the initial, reference pattern. In the case of visible light, this pattern is provided by the surface texture of the material or can easily be created by paint spraying. In the case of secondary electron images, the pattern was created by the deposition of particles $100 \mathrm{~nm}$ in size all over the specimen surface [8] or by scratching lines [7] or it was produced naturally by the microstructural features' differences in contrast [1,5]. Obviously, the accuracy and resolution of the measurements are very dependent on the nature of the reference pattern. For practical reasons, a random deposition of $\mathrm{nm}$ particles seems to be a suitable option because it is independent of the material and fine enough to measure local displacements at the scale of the microstructural features. 
In this paper, the potential of DIC is assessed for the first time to determine the displacement and strain fields at the microstructural level in a fiber-reinforced composite material at different magnifications. To this end, compression tests in a direction perpendicular to the fibers were carried out inside a scanning electron microscope. Secondary electron images were obtained at different magnifications during the test and processed using DIC. The accuracy of the measurements in terms of displacements and strains was determined by comparison with numerical simulations which took into account the actual distribution and diameter of the fibers within the microstructure. Finally, the potential applications and limitations of this strategy to study the deformation and fracture micromechanisms of composite materials is discussed.

\section{Material and experimental techniques}

Unidirectional laminates $\left([0]_{14}\right)$ were manufactured from preimpregnated sheets of E glass/ MTM 57 epoxy resin (Advanced Composite Group, UK). Rectangular panels of $350 \times 300 \mathrm{~mm}^{2}$ were heated at $3{ }^{\circ} \mathrm{C} / \mathrm{min}$ and consolidated at $120^{\circ} \mathrm{C}$ under $0.64 \mathrm{MPa}$ of pressure in an autoclave for $30 \mathrm{~min}$. They were cooled at the same rate of $3{ }^{\circ} \mathrm{C} / \mathrm{min}$ and the pressure was released at $80^{\circ} \mathrm{C}$. After manufacturing, the panels were inspected by ultrasound to ensure that they were free of delaminations or other defects. The nominal fiber volume fraction was $54 \%$. The elastic properties of both constituents were obtained from previous investigations [20], and can be found in (Table 1). They provide a stiffness ratio between fibers and matrix of $\approx 22$, and this large elastic contrast will lead to very inhomogeneous deformation fields in the composite microstructure.

Prismatic specimens of 3.2 (length) $\times 2.8$ (width) $\times 2.0$ (thickness) $\mathrm{mm}^{3}$ (Fig. 1) were machined from the unidirectional panels using a diamond wire cutting machine. This method ensures an almost perfect parallelism between the faces of the prismatic specimens, a critical condition to obtain an homogeneous stress distribution in the compression test. The specimens were subjected to uniaxial compression perpendicular to the fibers in the laminate within a scanning electron microscope (Zeiss EVO MA15). The compression axis was parallel to the longest dimension of the specimen and load was applied using two parallel steel compression plates mounted on a micro-electro-mechanical testing machine (Kammrath-Weiss Tensile/Compression Stage). The specimen-plate friction was minimized by coating the upper and lower specimen surfaces with vacuum wax. Compression tests were carried out at a cross-head speed of $1 \mu \mathrm{m} / \mathrm{min}$. The load and cross-head displacement were continuously recorded during the test using the load cell and displacement transducer of the mechanical testing machine. Secondary electron micrographs with $768 \times 1024$-pixel resolution were taken of the central area of the specimen at different magnifications in the range $70-6000 \times$ in the undeformed configuration as a reference. The central area of the specimen is the region around the center of the sample, where the solid rigid displacements are supposed to be minimum. Once the test began, it was interrupted periodically and held at a fixed displacement until full stress relaxation of the specimen was attained The relaxation produced small reduction of the applied load, always below $5 \%$, and took place in a few minutes. Secondary electron micrographs were then taken of the central area of the specimen with the same resolution and magnification. They were

Table 1

Elastic properties of MTM57 epoxy matrix and E-glass fibers.

\begin{tabular}{lll}
\hline & $E(\mathrm{GPa})$ & $v$ \\
\hline E-glass fibers & 74 & 0.20 \\
MTM57 epoxy matrix & 3.35 & 0.35 \\
\hline
\end{tabular}

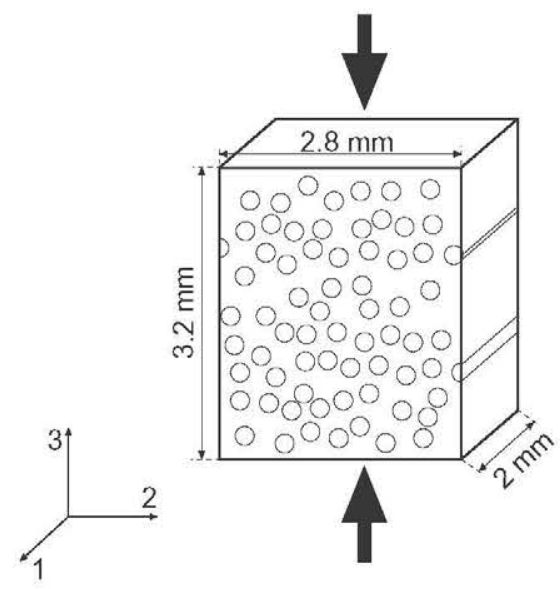

Fig. 1. Schematic of the compressive specimen. Fibers have been enlarged two show their orientation within the specimen.

used as input for full-field measurements using the Vic2D digital image correlation software (VicSNAP, Correlated Solutions, Inc.).

The surface preparation of the specimens was crucial to obtain good results using DIC. The microstructure of the material was first revealed by polishing a section perpendicular to the fibers using $\mathrm{SiC}$ paper to a 1000 grit finish followed by a diamond slurry up to $1 \mu \mathrm{m}$ grain size. A speckle pattern was created on this surface by depositing submicron alumina particles from a suspension containing $1 \mathrm{wt} \%$ of $\mathrm{Al}_{2} \mathrm{O}_{3}$ in propanol. The submicron powders tended to agglomerate leading to particle clusters whose size was similar to the fiber diameter but this effect was eliminated by adding iodine to the suspension as a dispersant. A typical pattern is found in the scanning electron micrograph of Fig. 2, showing an homogeneous dispersion of submicron alumina particles on the specimen surface. The maximum greyscale level in the image was generated by the alumina particles, and the average intensity was 130 (within the range 0-255). Meanwhile, the background microstructure showed an average intensity of 63 with a minimum greyscale level of 36 . Thus, the contrast provided by this speckle pattern was good enough to correlate the displacement fields.

\section{DIC in heterogeneous materials}

DIC is a non-contacting optical technique to measure the displacement field on the surface of a specimen at different stages

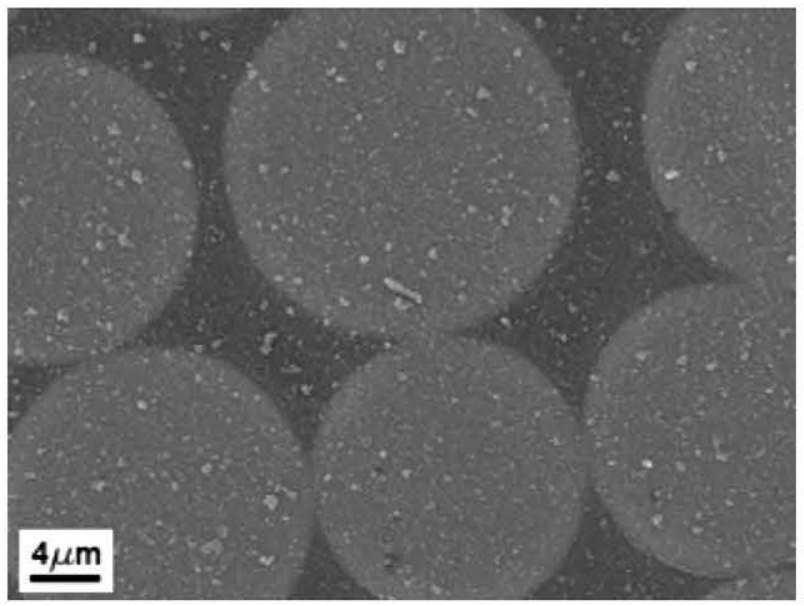

Fig. 2. Scanning electron micrograph of the specimen surface showing the homogeneous dispersion of submicron alumina particles. 
during deformation. The full strain field on the surface can be obtained afterwards as the derivative of the displacement field. The foundation of the technique is very simple: the displacement field is computed by tracking the distribution of grey intensity on the specimen surface in images acquired at different stages upon loading [12].

The position of an arbitrary pixel $i$ of the image in the undeformed specimen can be expressed in terms of its coordinates $\mathbf{X}_{i}$ in the reference configuration. This pixel will be displaced to a new position given by the coordinates $\mathbf{x}_{i}$ in the deformed configuration. Obviously, both coordinates are related by

$\mathbf{x}_{i}=\mathbf{X}_{i}+\mathbf{U}_{i}$

where $\mathbf{U}_{i}$ is the displacement vector. Let $G(\mathbf{X})$ and $g(\mathbf{x})$ be the intensity of the gray level in each pixel of the images corresponding to the undeformed and deformed configurations, respectively. If it is assumed that the intensity pattern deforms but it does not change its local value during deformation (i.e., $g(\mathbf{x})=G(\mathbf{X}+\mathbf{U})$ ), it is possible to track the displacement of every point of the specimen surface by identifying the displacement field which minimizes a residual function $C$ [18] over the whole region of interest, where $C$ is expressed as

$C(\mathbf{X}, \mathbf{x})=1-\frac{\sum G(\mathbf{X}) g(\mathbf{x})}{\sqrt{\sum G(\mathbf{X})^{2} \sum g(\mathbf{x})^{2}}}$

This minimization problem is, however, too complex to be solved in a general case and it has to be simplified by introducing two main approximations. Firstly, the displacement of an arbitrary point is obtained from the evaluation of the gray intensity in its neighborhood. This set of pixels around the central one is called the subset. Secondly, the deformations are constant within this subset. Under these assumptions, the coordinates $\mathbf{X}_{i}$ and $\mathbf{x}_{i}$ of a point of the subset in the reference and deformed configurations can be related with the coordinates of the central point of the subset $\left(\mathbf{X}_{c}\right.$ and $\mathbf{x}_{c}$ ) by

$\mathbf{x}_{i}=\mathbf{X}_{c}+\mathbf{U}_{c}+\nabla(\mathbf{U})\left(\mathbf{X}_{i}-\mathbf{X}_{c}\right)$

where $\nabla(\mathbf{U})$ stands for the constant displacement gradient within the subset. Therefore, the subset is limited to capture homogeneous deformations. Both $\mathbf{U}_{c}$ and $\nabla(\mathbf{U})$ are obtained from the iterative minimization of the correlation function $C$ (Eq. (2)) within the subset. Once the displacement of the central pixel has been obtained, the subset is shifted along $x$ - and $y$-directions to calculate the next point. The shift of the subset is determined by the step size, which defines how many pixels the subset was moved. Finally, the strains are computed according to the Lagrangian approach by taking the numerical derivative of the displacement field with a spacing defined by the strain window. A detailed overview of displacement and deformation measurements through digital image correlation, and its application to practical cases can be found in Ref. [17].

The application of DIC to microscopic images obtained in electron or atomic force microscopes does not present any particular difficulty insofar as the image contains a suitable speckle pattern $[1,4,5,8,22,24]$. However, DIC is a fuzzy measuring technique since it averages the displacement over the subset window [23] and the strains are computed as the derivative of the displacements on another window. This is not a problem to capturing non-uniform fullfield deformations in homogeneous materials, but limits the accuracy of the technique in the case of heterogeneous materials with strong contrast in the elastic properties of the phases if the resolution of the full-field measurements is of the order of the heterogeneity size. This limitation is due to the presence of strong deformation gradients at the interface between phases, whose evaluation may be compromised by the necessity of averaging displacements and deformations over a certain window. Thus, although DIC is a well-established technique for obtaining the strain fields at the macroscopic scale in composites [2,20,21] and fabrics $[9,10,14]$, this approach has never been used -to the authors' knowledge- at the scale of the fibers due to the differences in stiffness between matrix and fibers.

The rigorous extension of DIC to non-homogeneous materials is not straightforward. Recent attempts were focused on the application of an interpolation technique based on the X-FEM method to account for the discontinuities in the strain field caused by microcracks [13]. The methodology is not fully established, however, and neither is it able to deal with discontinuities due to phases with different elastic properties. So, we have preferred to assess the potential of standard DIC techniques and algorithms to capture the displacement and strain fields at the micron level in a standard fiber-reinforced composite material. In order to optimize the DIC parameters and to assess the limitations, a benchmark problem has been studied in which the exact elastic response can be computed. The problem deals with the uniaxial deformation of a representative volume element (RVE) of a model composite microstructure containing circular elastic inclusions embedded in a continuous matrix. Fiber and matrix elastic properties were those of glass fibers and epoxy resin in Table 1, and the spatial distribution of the fibers in the RVE is shown in Fig. 3a. Uniaxial tension of the RVE was simulated applying a horizontal displacement on the right hand side while the left hand side of the RVE was fixed. The bottom and top boundaries of the RVE were stress free. The exact solution to the problem was obtained using the finite element (FE) method
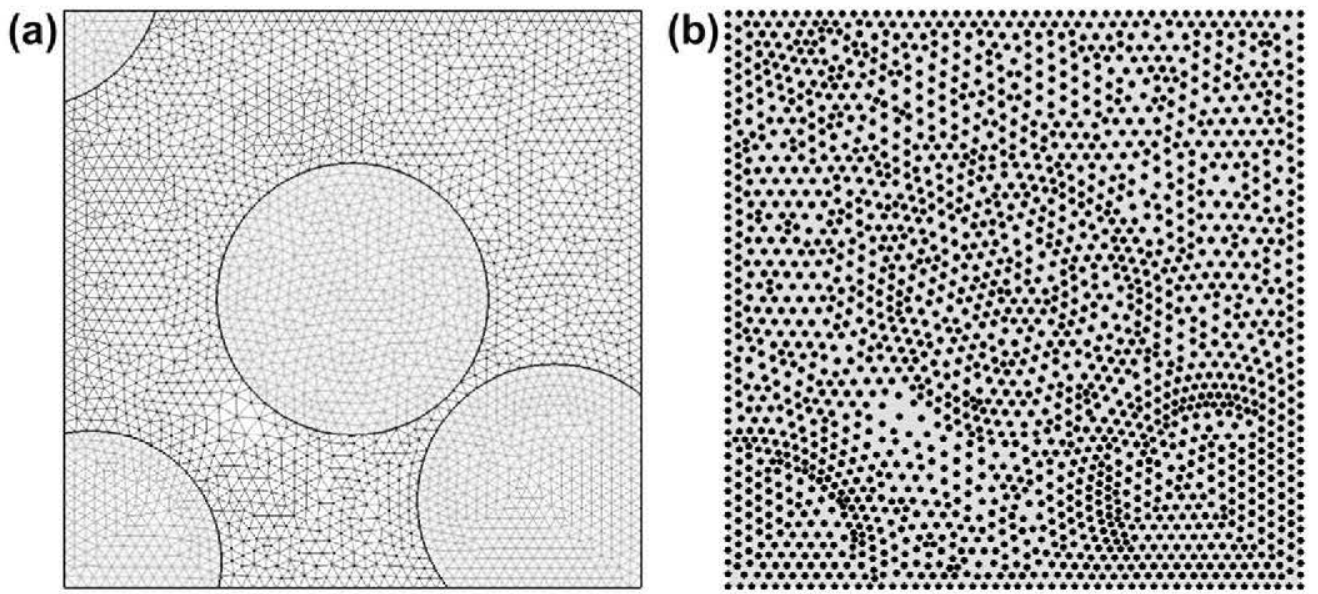

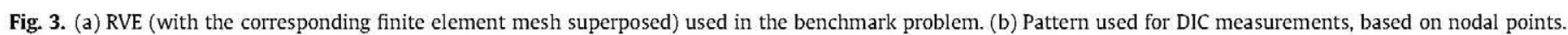


with a very fine mesh, and the displacement and strain fields derived from the simulation were used as a reference to check the accuracy of DIC.

Images obtained from the FE simulations in the initial and deformed configurations were used as input for DIC. A step size of 5 pixels was maintained constant during the correlations, and the search for the optimum DIC parameters was focused in the subset and strain window size. The pattern used consisted of black dots, corresponding to each nodal point, over a grey background (Fig. 3b). The contrast of the computer-generated images was better than the one provided by the scanning electron micrographs. However, the size and the distribution of the black dots were comparable to the alumina particles on the specimen surface. Thus, we could use subset and strain window sizes similar to those used later for the SEM images. The contour plot of the displacements in the loading (horizontal) direction obtained from the FE analysis and from DIC with different subset sizes are plotted in Fig. 4 . They show very good agreement, and the selection of the subset size did not significantly influence the results although transient high frequency oscillations began to be noticeable for very small subset sizes (Fig. 4b). Thus, these results show that the presence of heterogeneities does not impair the ability of DIC to accurately capture the displacement fields.

The contour plots for the horizontal strain field provided by the FE simulations and DIC are plotted in Fig. 5. The results of DIC in the figure were obtained using a subset size of $0.182 \mathrm{D}$ and strain windows of $0.0179 D$ and $0.182 D$, where $D$ is the fiber diameter ( 0.5 the length of the RVE). At first glance, the strain fields obtained by DIC are in good agreement with the exact solution given by the FE analysis in matrix and fiber regions far away from the interface. However, the fuzzy nature of DIC makes it impossible to capture the sharp strain gradients at the fiber-matrix interface and it is necessary to optimize the parameters of DIC (namely the subset size and strain window) to enhance the accuracy of the approximation. To this end, the horizontal strains computed by FE and DIC were plotted in Fig. 6 along a diagonal path from the upper left to the lower right corner of the RVE. DIC results in Fig. 6a show the influence of the subset size at a constant value of the strain window (equal to $0.0536 \mathrm{D}$ ), while the curves in Fig. $6 \mathrm{~b}$ present the influence of the strain window for a constant subset size of $0.182 \mathrm{D}$. The FE solution shows zones in the strain path in which the strain is very low and constant, which correspond to the stiff fibers. Matrix regions between fibers present much higher strains with very sharp gradients close to the fiber interface. DIC was able to accurately compute the strain in the center of the fibers as well as in the matrix regions far away from the fiber interface. However, significant differences appeared near to the interface between both materials because of the discontinuity in the strain field. DIC obtains the strains by taking the derivate of the displacements over a small strain window without considering the phase in which the pixels are located and the discontinuity at the interface is smoothed out.

The curves in Fig. 6a show that subset size did not substantially modify the strain profiles along the path and this was expected because this parameter only influences the magnitude of the displacement field, which was very accurate in the whole range of subset sizes $(0.104-0.361 D)$ used in this investigation. However, the strain window did modify the strain values along the path. The smaller the window, the higher the strain gradients, and thus DIC was able to capture the variations in strain near the interfaces in greater detail. Nevertheless, if the strain window was too small, high frequency oscillations appeared in the solution, leading to unrealistic oscillations of the strains within each phase. For this reason, the optimum window size should be a compromise between a small one that provides a more accurate "local" field value, and a large one that gives a smoother solution. In the case of the (a)
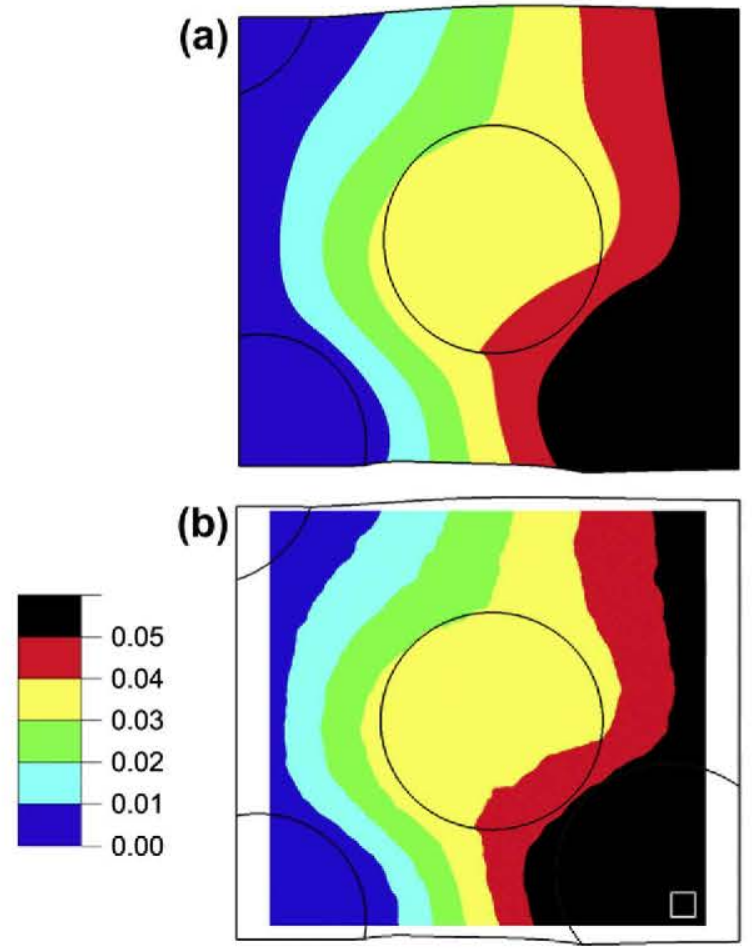

(c)

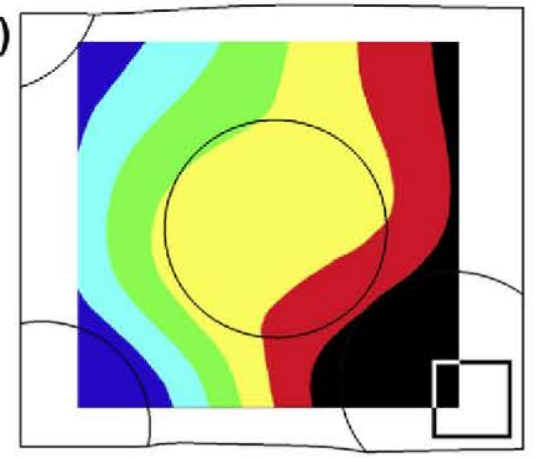

Fig. 4. Contour plots of the horizontal displacement field in the benchmark problem. (a) Reference solution provided by FE simulations. (b) DIC field with a subset size of $0.104 \mathrm{D}$ (29 pixels), the subset window is displayed in the image. (c) DIC field with a subset size of $0.361 \mathrm{D}$ (103 pixels). The displacements are normalized by the length of the unit cell. (For interpretation of the references to color in this figure legend, the reader is referred to the web version of this article.)

model RVE for composite materials, the optimum window size was around $0.05 \mathrm{D}$ which corresponds to the minimum strain window which provides logical and realistic strain fields with the minimum possible noise. With this window size, it was able to accurately reproduce the average strains in each phase and only failed to capture the strain in matrix ligaments between very close fibers.

\section{Experimental results}

Transverse compression tests were performed using the microelectro-mechanical testing machine inside the scanning electron microscope as indicated above. The specimens were elastically strained up to $660 \mathrm{~N}$ of load (corresponding to an average compressive stress of $\approx 120 \mathrm{MPa}$ ) and there was no evidence of damage by interface decohesion. Micrographs with $768 \times 1024$ pixels resolution of a region of interest (ROI) at the center of the specimen were obtained at different magnifications and used as input for DIC analyses. 

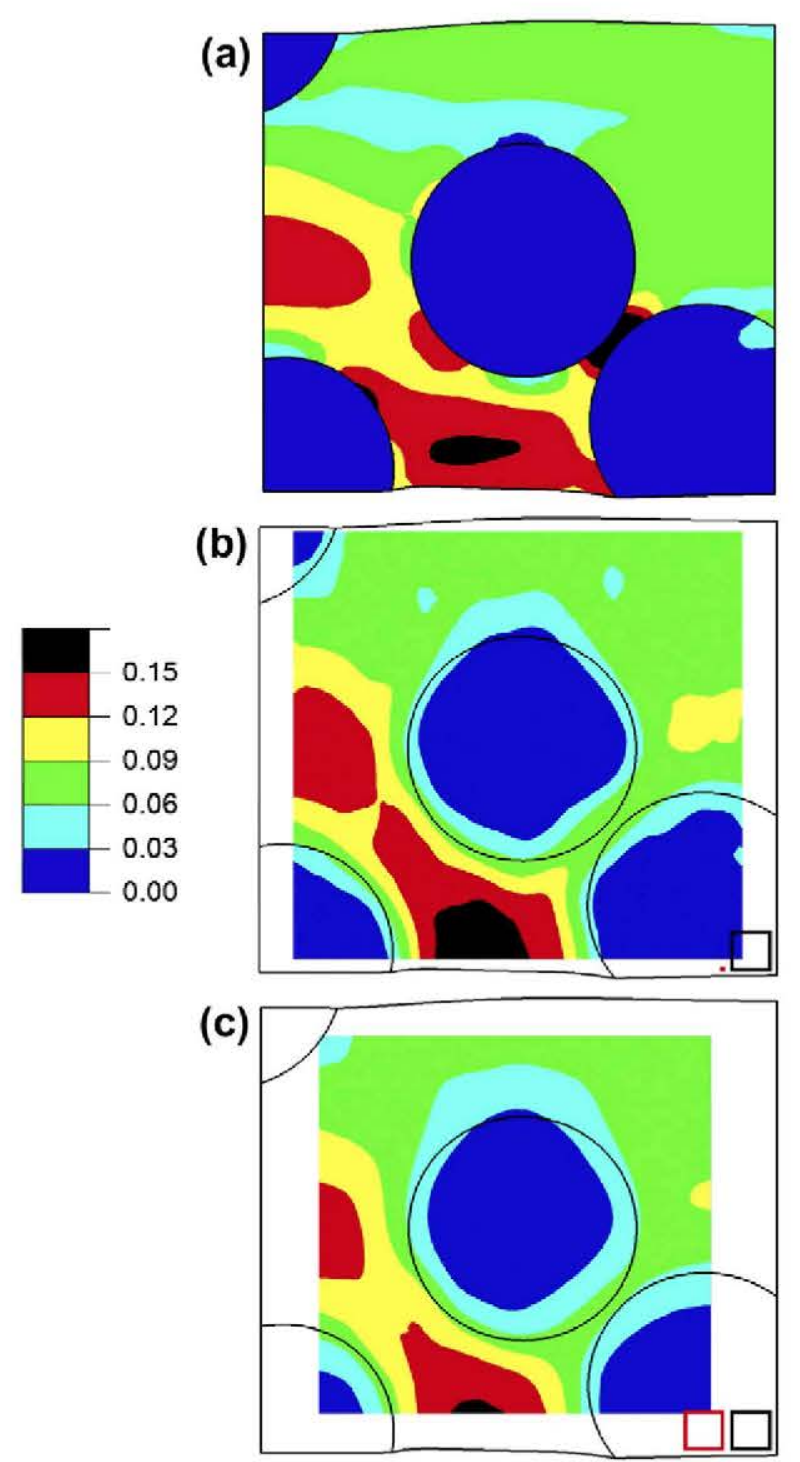

Fig. 5. Contour plots of the horizontal strain field in the benchmark problem. (a) Reference solution provided by FE simulations. (b) DIC field obtained with a subset size of $0.182 \mathrm{D}$ ( 51 pixels) and a strain window of $0.0179 \mathrm{D}$ ( 5 pixels), the subset (black) and the strain (red) windows are shown in the images. (c) DIC field obtained with a subset size of $0.182 \mathrm{D}$ and a strain window of $0.182 \mathrm{D}$. (For interpretation of the references to color in this figure legend, the reader is referred to the web version of this article.)

\subsection{Low magnification $(250 \times)$}

The alumina nanoparticles could not be resolved on the specimen surface at this magnification and the contour of the glass fibers acted as speckle pattern for DIC. The evaluation of the displacements and strains was carried out with a subset size of 50 pixels and a strain window of 15 pixels. This subset size corresponds to approximately $50 \mu \mathrm{m}(2.5 \mathrm{D})$, while the window strain stands for $0.75 D$. These large sizes for the subset and window strain limited the resolution of DIC, but it did not make sense to reduce their size because of the lack of an appropriate speckle pattern to detect the strain gradients between fibers.

The displacement field along the vertical (loading) direction obtained from a micrograph of the ROI at $250 \times$ is shown in Fig. 7a. Despite local fluctuations, the iso-displacement lines were almost perpendicular to the loading axis, corresponding to an homogeneous stress state characteristic of uniaxial compression.
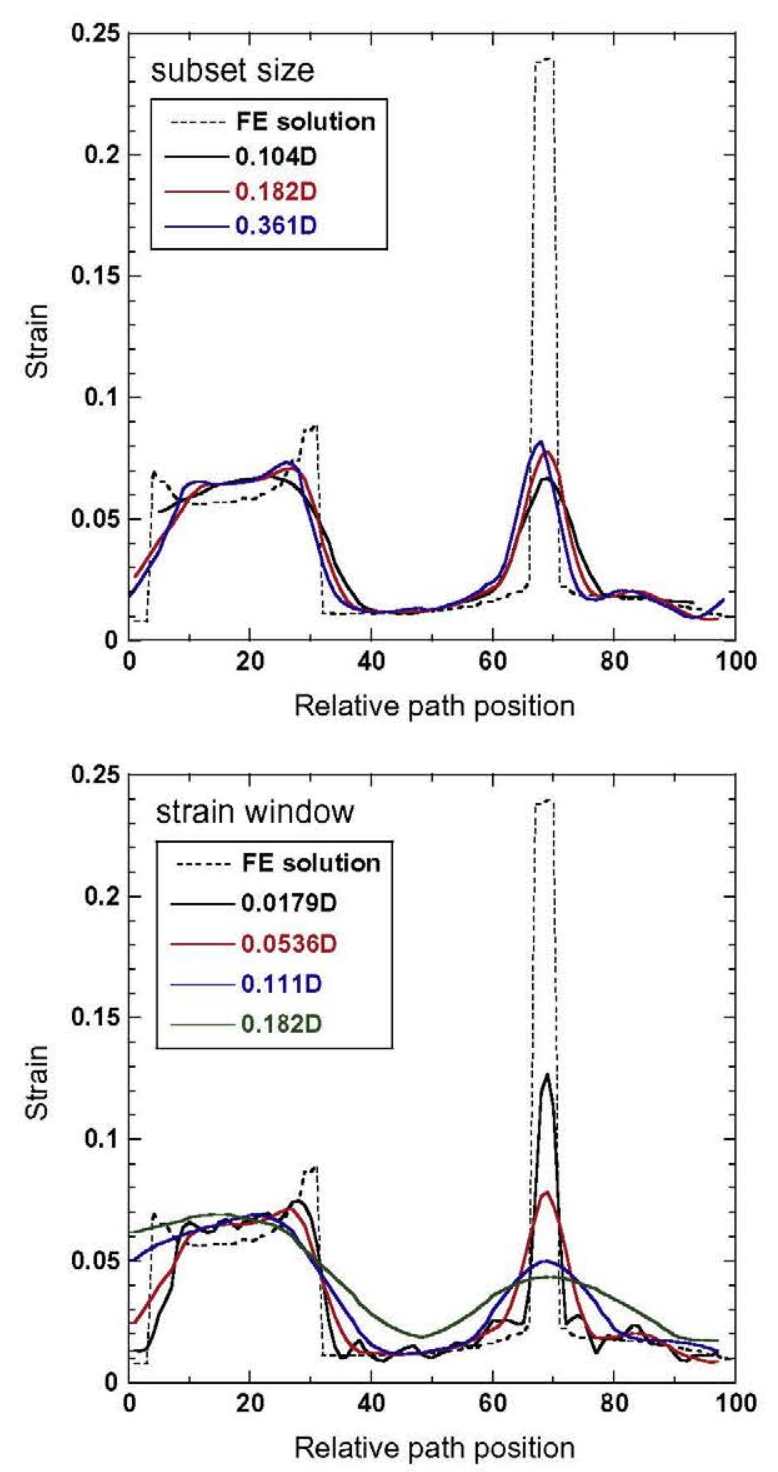

Fig. 6. Horizontal strains along a diagonal path from the upper-left to the bottomright corner of the RVE. (a) Influence of the subset size on DIC results. The strain window was $0.0536 \mathrm{D}$. (b) Influence of the strain window on DIC results. The subset size was $0.0182 \mathrm{D}$. (For interpretation of the references to color in this figure legend, the reader is referred to the web version of this article.)

These results corroborate the adequacy of the experimental setup to carry out uniaxial compression tests.

The contour plot of the compressive strain along the loading axis is plotted in Fig. 7b on top of the fiber distribution in the composite lamina. The large subset size as compared to the fiber diameter and the fact that the fiber contours acted as the speckle pattern at this magnification completely smoothed out the strain jumps associated with the fiber/matrix interface but the plot shows noticeable fluctuations in the strain throughout the ROI. They correspond to local non-homogeneities in fiber volume fraction. The local reinforcement volume fraction of three representative regions of the microstructure was computed within square windows of $100 \mu \mathrm{m}$ (Fig. 7b). The calculated values of the volume fraction at V1, V2 and V3 was $0.47,0.52$ and 0.63 , respectively. Strain was localized in matrix rich regions while it was minimum in zones containing clusters of highly packed fibers and only the non-homogeneities associated with fiber clusters were detected. This effect is illustrated by splitting the strain distribution into fiber and matrix areas and computing the probability density plots 

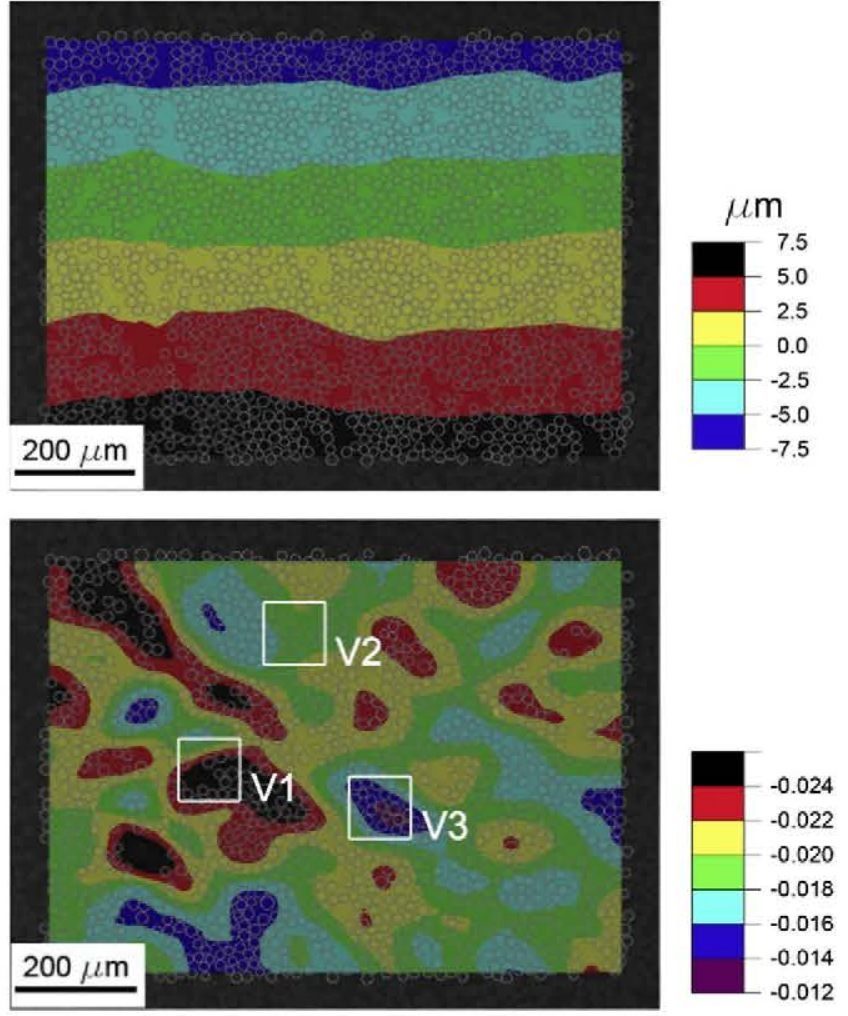

Fig. 7. (a) Vertical displacement field obtained using DIC in a micrograph at $250 \times$. (b) Compressive strain in the vertical (loading) direction obtained from (a). The fiber contours were artificially highlighted during post-processing of the images. (For interpretation of the references to color in this figure legend, the reader is referred to the web version of this article.)

individually (Fig. 8). They were not distinguishable from the overall one, indicating that the short range elastic interactions between individual fibers were not captured and were averaged over the subset size. The presence of matrix-rich and fiber-rich regions in the ROI is visualized in the width of the bell curve for the probability density plot which is mainly controlled by the contrast between elastic moduli of fibers and matrix (of the order of 22 for a typical glass/epoxy composite). The larger the contrast, the higher the difference in the elastic properties between the clustered regions and the resin rich regions and the greater the width of the bell curve of the probability density plot. It should finally be noted that the average strain value of the composite (as obtained from the probability density plot) was $\bar{\epsilon}=-1.786 \%$, which corresponds to an elastic modulus of the composite of $6.6 \mathrm{GPa}$. This value for the transverse elastic modulus of the glass-fiber composite is accurate, according to previous experimental results [11]

\subsection{High magnification $(2000 \times$ and $6000 \times)$}

The DIC analysis of the micrographs obtained at higher magnification was carried out with a subset size of 50 pixels and a strain window of 15 pixels. The subset size of 50 pixels corresponds to approximately $6.5 \mu \mathrm{m}(0.325 D)$ and $2.2 \mu \mathrm{m}(0.11 D)$ for the magnification factors of 2000 and 6000 , respectively, while the strain window of 15 pixels stands for $0.0975 D$ and $0.033 D$. According to the analysis in Section 3, these sizes were chosen to optimize the results provided by DIC at the highest magnification $(6000 \times)$ while they were expected to provide reasonable results for the average strain in the composite at $2000 \times$.

The vertical displacement fields obtained with DIC on micrographs obtained at $2000 \times$ and $6000 \times$ are plotted in Fig. 9a and

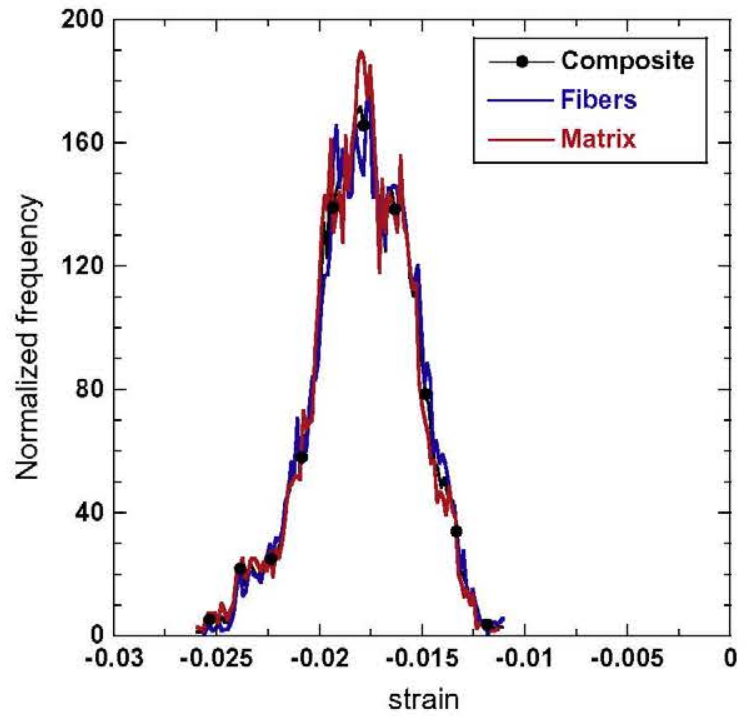

Fig. 8. Probability density plots of the strain in the loading direction for fiber regions, matrix regions and the composite obtained from micrographs at $250 \times$ analyzed using DIC. (For interpretation of the references to color in this figure legend, the reader is referred to the web version of this article.)
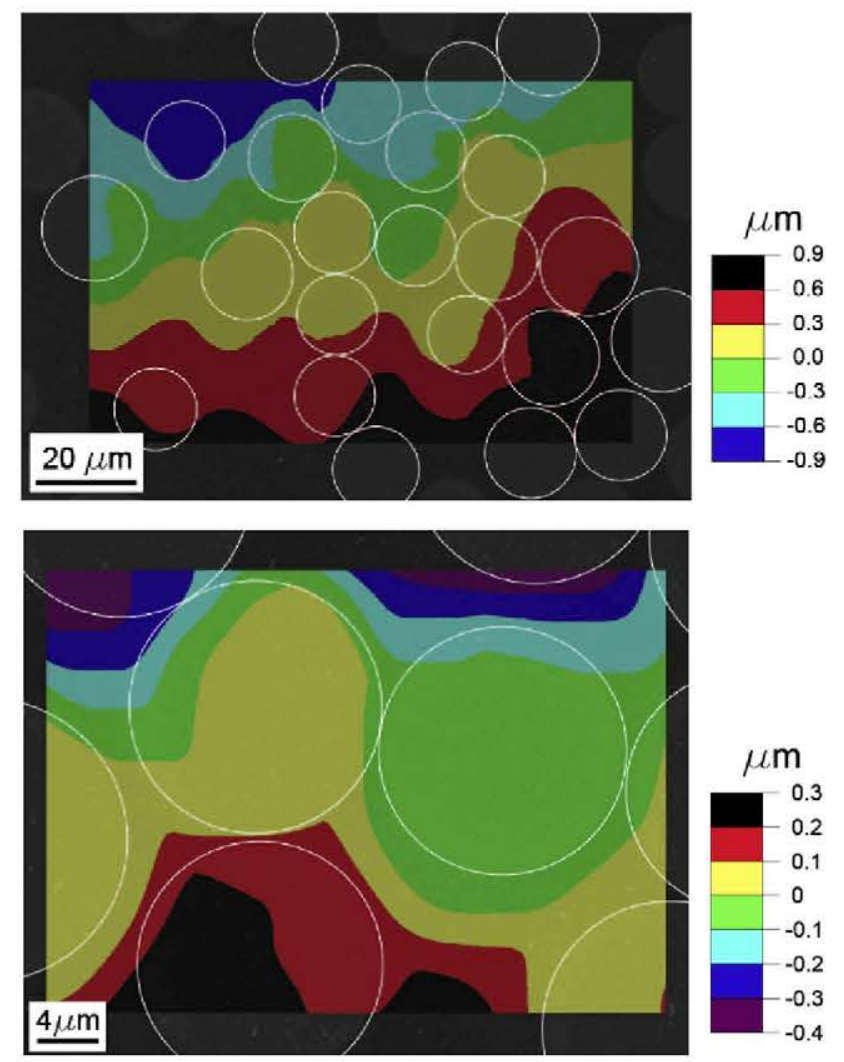

Fig. 9. (a) Vertical displacement field obtained using DIC in a micrograph at $2000 \times$. (b) Vertical displacement field obtained using DIC in a micrograph at $6000 \times$. Loading axis is vertical. (For interpretation of the references to color in this figure legend, the reader is referred to the web version of this article.)

b, respectively. The non-homogeneities in the displacement fields induced by the presence of matrix and fibers are resolved to the scale of the fibers at both magnifications. The corresponding vertical strain fields obtained by taking the derivative of the displacement fields are plotted in Fig. $10 \mathrm{a}$ and b for $2000 \times$ and $6000 \times$, 
respectively. At first glance, it is obvious that the strain fields obtained with DIC qualitatively reproduced the expected pattern: strains in the fibers were much smaller than those found in the matrix and maximum strain concentrations were found in the matrix between fibers aligned in the (vertical) loading direction, the applied strain being mainly accommodated by the matrix ligaments between the fibers. The presence of small longitudinal tensile strains should also be noted, very close to the resolution of DIC, which appeared in fiber pairs very close to each other in the direction perpendicular to the loading axis. They were generated probably as a result of the development of large contact stresses between individual fibers upon deformation.

DIC was thus able to discriminate between fiber and matrix strains at these magnifications and this is quantitatively demonstrated in Fig. 11, which shows the probability density plots for the matrix and fibers regions as well as for the overall composite from the micrographs at $6000 \times$ magnification. The shape and intensity of the probability density functions for each phase were different from the corresponding plots obtained at $250 \times$ (Fig. 8). Fibers were characterized by a narrow peak which stands for an homogeneous strain field within the phase, while the matrix showed a wide distribution indicative of a very non-homogeneous strain distribution within the phase. The probability density functions obtained from the micrographs at $2000 \times$ are equivalent and are not plotted for the sake of brevity.

The average composite strain $(\bar{\epsilon})$ as well as the average strains in each phase $\left(\bar{\epsilon}_{f}\right.$ and $\left.\bar{\epsilon}_{m}\right)$ were obtained from the probability density plots and are shown in Table 2 . The average composite strain at $2000 \times$ was very close to that obtained at $250 \times$ while the result obtained at $6000 \times$ was slightly lower. This difference was not attributed, however, to the inaccuracy of DIC but to the actual fluc-
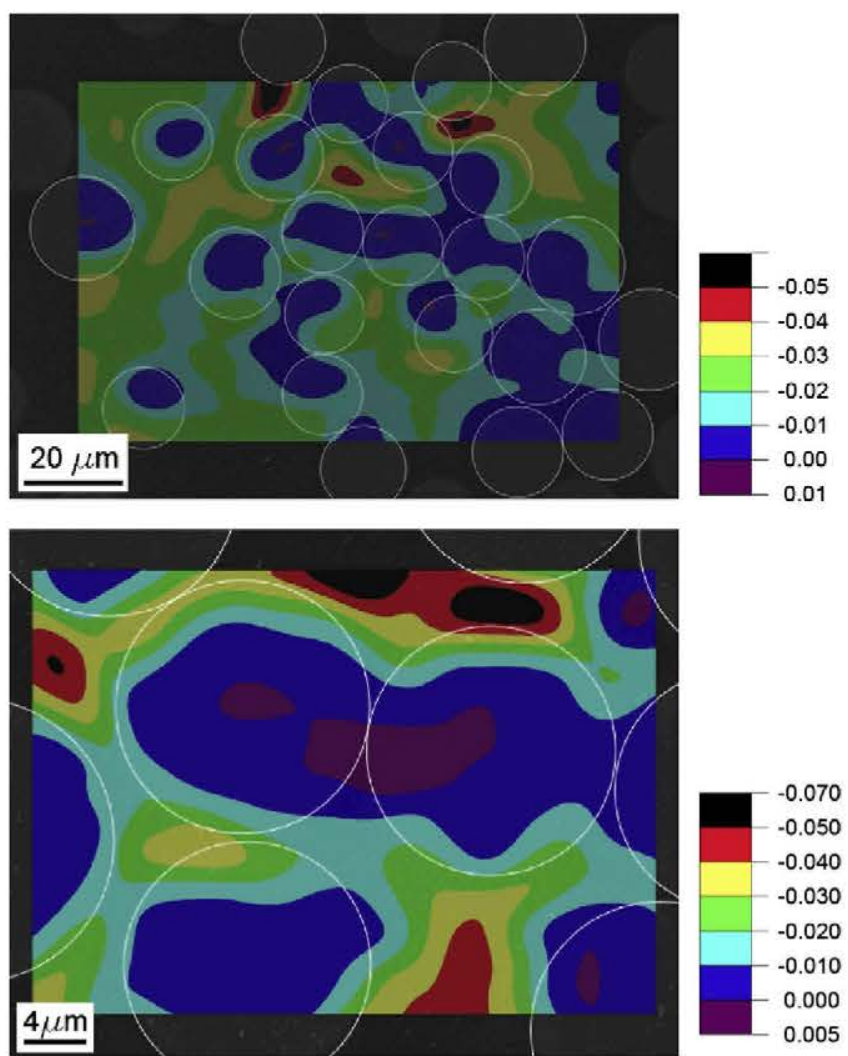

Fig. 10. (a) Vertical strain field obtained using DIC in a micrograph at $2000 \times$. (b) Vertical strain field obtained using DIC in a micrograph at $6000 \times$. Loading axis is vertical. (For interpretation of the references to color in this figure legend, the reader is referred to the web version of this article.)

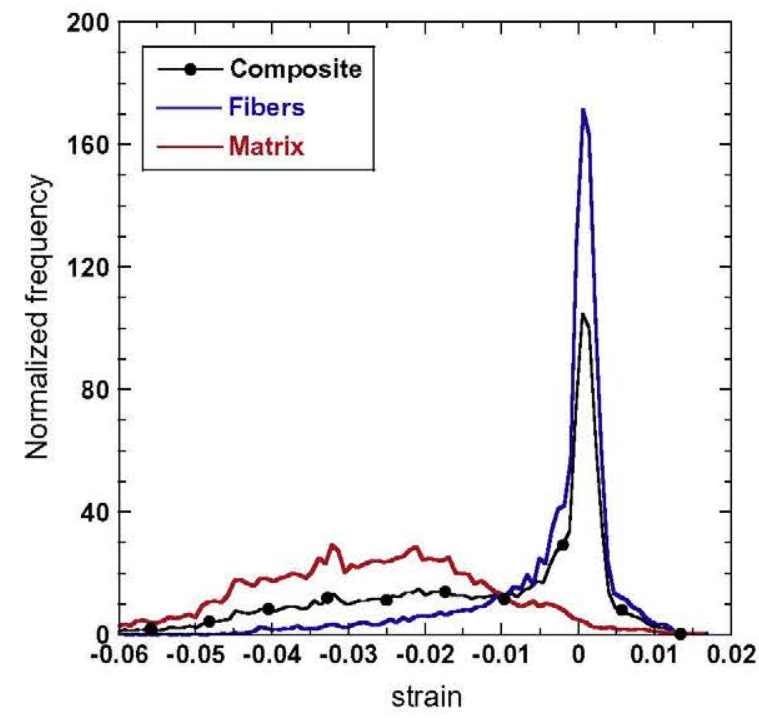

Fig. 11. Probability density plots of the strain in the loading direction for fiber regions, matrix regions and the composite obtained from micrographs at $6000 \times$ analyzed using DIC. (For interpretation of the references to color in this figure legend, the reader is referred to the web version of this article.)

Table 2

Average strains in the loading direction obtained by DIC and FE simulations.

\begin{tabular}{llll}
\hline & $\bar{\epsilon}$ & $\bar{\epsilon}_{f}$ & $\bar{\epsilon}_{m}$ \\
\hline $\operatorname{DIC}(2000 \times)$ & -0.0169 & -0.0094 & -0.0237 \\
$\operatorname{DIC}(6000 \times)$ & -0.0148 & -0.0089 & -0.0237 \\
$\operatorname{FE}(2000 \times)$ & -0.0175 & -0.003 & -0.0362 \\
$\operatorname{FE}(6000 \times)$ & -0.0145 & -0.0032 & -0.0363 \\
\hline
\end{tabular}

tuations in the local reinforcement volume fraction when the number of fibers in the ROI is small. In fact, the reinforcement volume fraction in the ROI at $6000 \times$ was $61 \%$ while the average reinforcement volume fraction in the composite was only $54 \%$. The average strain values in the matrix and the fibers obtained at $2000 \times$ and $6000 \times$ were similar but the accuracy of these values is questionable due to the limitations of DIC in the presence of strain discontinuities created by the mismatch in elastic properties, as demonstrated in Section 3. In order to ascertain the overall validity of these results, they have to be compared with benchmark results.

\section{Numerical model}

Although the results presented above show that DIC is able to qualitatively capture the non-homogeneous strain field which develops in composite materials at the micron scale, it is also evident from these plots that the fuzzy nature of the technique leads to errors in the strain measurement near to the fiber/matrix interface. In order to quantify these errors, the actual displacement and strain fields corresponding to the ROI at $2000 \times$ (Figs. 9a and 10a) and $6000 \times$ (Figs. $9 \mathrm{~b}$ and $10 \mathrm{~b}$ ) were determined by means of computational micromechanics. This technique has been successfully applied in the past to modeling the deformation and fracture micromechanisms of composites at the micron scale and it is based on the finite element (FE) analyses of a representative element of the material in which the microstructural details (fibers and matrix) are explicitly represented $[6,16,19]$.

In this particular case, the real microstructures used for DIC (Figs. 9 and 10) were exactly replicated in a two-dimensional finite element model. The matrix and fibers were automatically meshed 

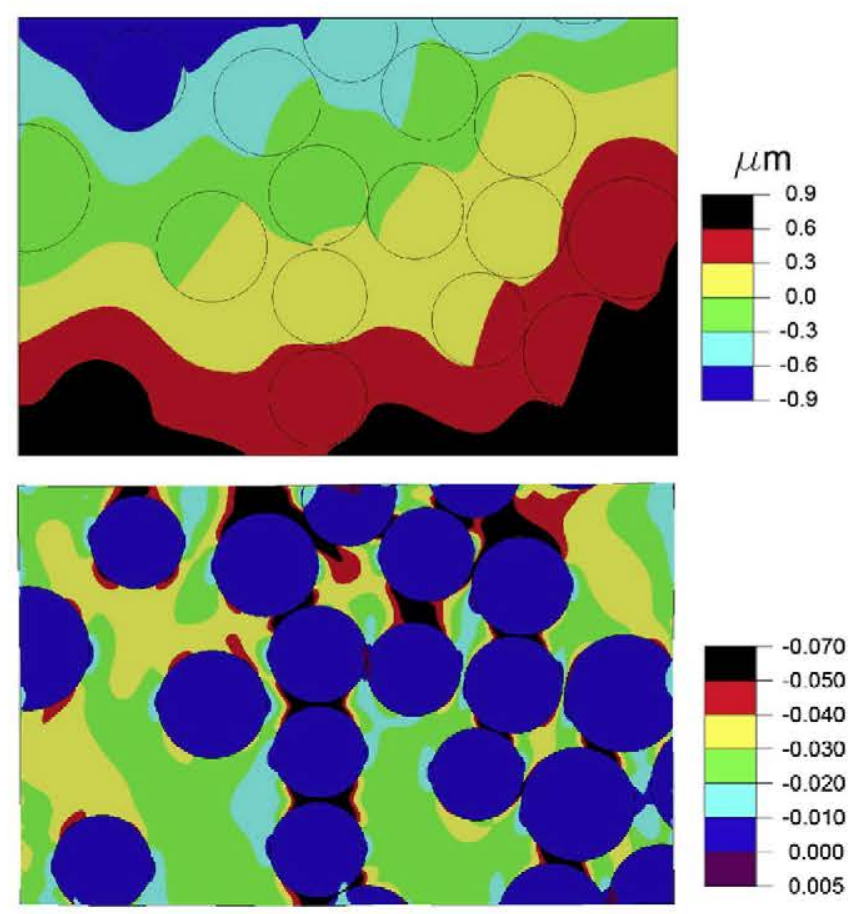

Fig. 12. (a) Vertical displacement field obtained from the numerical simulation of a micrograph at $2000 \times$. (b) Vertical strain field obtained from the numerical simulation of a micrograph at $2000 \times$. Loading axis is vertical. (For interpretation of the references to color in this figure legend, the reader is referred to the web version of this article.)

using 6-node isoparametric modified triangles (CPE6M in Abaqus Standard) with integration at three Gauss points and hourglass control. Special care was taken to generate a very fine and homogeneous mesh at the ligaments between adjacent fibers to resolve the large deformation gradients which appear in these regions upon deformation. The matrix/fiber interfaces were assumed to be perfect and damage by decohesion was not included in the model in agreement with the experimental observations. Matrix and fibers behaved as linear, elastic and isotropic solids, whose elastic constants can be found in Table 1. The actual displacements in the longitudinal and vertical directions measured by DIC on the external boundary of the model were applied to the finite element model. This ensured the displacement continuity with the composite outside of the region analyzed and introduced the deformation patterns experimentally observed into the model. The real problem is truly three dimensional and cannot be exactly simulated under plane strain or plane stress conditions. However, the exact response is in between both cases and simulations in the two limiting conditions were carried out in Abaqus/Standard. The differences between them were negligible for the displacement and strain fields in the $x y$-plane.

The contour plots of the vertical displacement and of the vertical strain obtained from the finite element simulation of the microstructure at $2000 \times$ are shown in Fig. $12 \mathrm{a}$ and b, respectively. They can be compared with the DIC results in Figs. 9a and 10a. The displacement field provided by the numerical simulations was qualitatively equivalent to the experimental one obtained by DIC, demonstrating the potential of this technique to reveal the displacement patterns at the microscopic scale. Both strategies clearly showed that fibers acted as pinning points and that the iso-displacement lines were highly curved around the fibers. A better quantitative comparison can be obtained by plotting the magnitude of the vertical displacement field along a diagonal line from the lower left to the upper right corner (Fig. 13a). The general
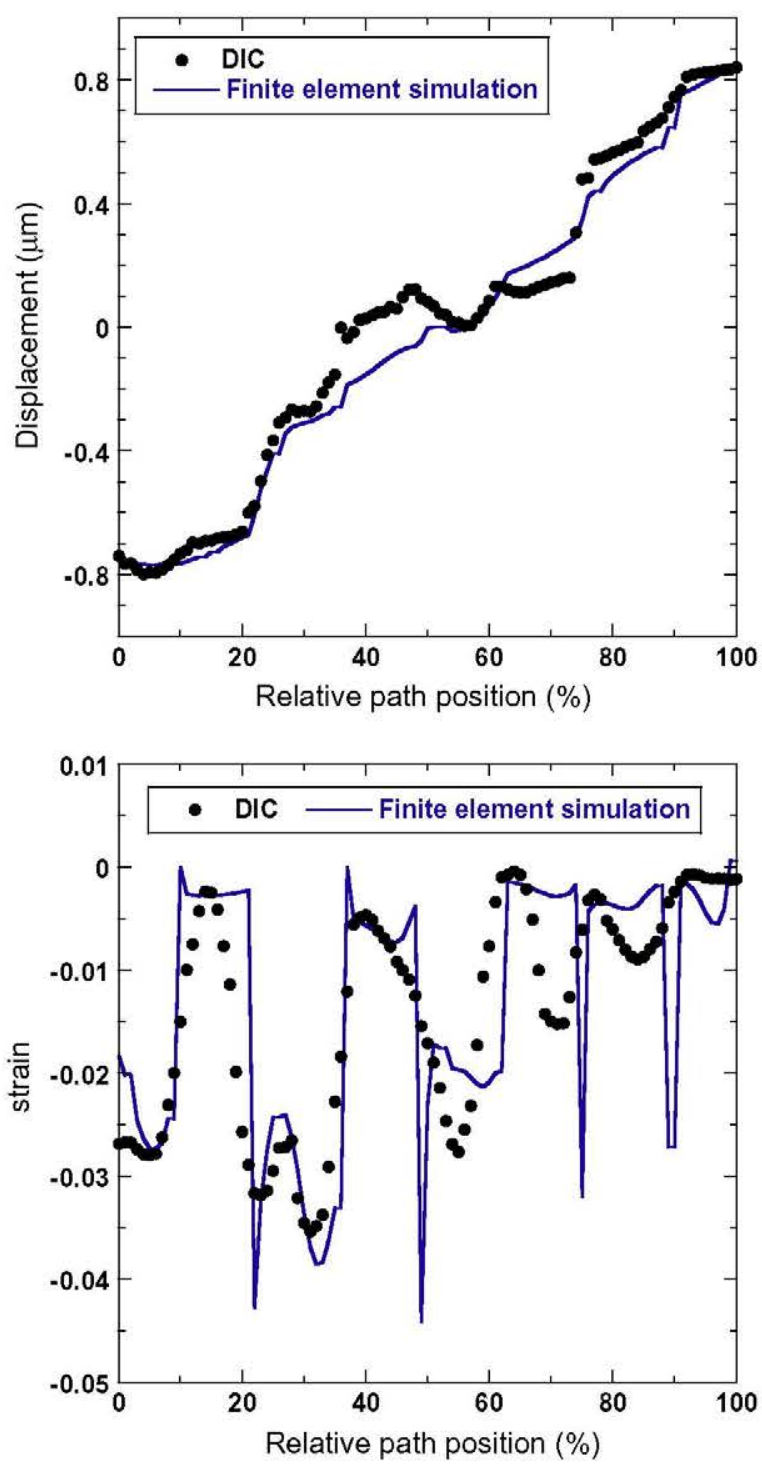

Fig. 13. Comparison of digital image correlation measurements at $2000 \times$ and the corresponding finite element results along a diagonal path from the bottom left to the upper right corner of the ROI. (a) Vertical displacement. (b) Vertical strain. (For interpretation of the references to color in this figure legend, the reader is referred to the web version of this article.)

agreement between the experiments and simulations is fairly good but there were noticeable differences at the local level in the gradients near to the interfaces.

The comparison of the numerical and experimental strain fields (Figs. $12 \mathrm{~b}$ and $10 \mathrm{a}$ ) follows the same trends although the differences were even more marked. Generally speaking, agreement is good and the strain field trends are well captured even with the relatively large subset size used in this study ( 50 pixels, which corresponds to $\approx 6.5 \mu \mathrm{m}$ at $2000 \times$ and $\approx 2.2 \mu \mathrm{m}$ at $2000 \times$ ), which are of the order of magnitude of the fiber diameter. Nevertheless, the strain fields given by DIC within the fibers presented unrealistic gradients, while the finite element simulations showed that the strain within the fibers was fairly constant. Obviously, these differences come about as a result of the smoothing of the large strain gradients at the fiber/matrix interface into the fibers due to the DIC subset size. A more detailed quantification of this effect can be found in Fig. 13b, in which the vertical strain is plotted along a diagonal line from the lower left to the upper right corner of the ROI. This plot shows the limitation of DIC to capture the sharp 
strain jumps at the fiber/matrix interface and the modification of the average strain in the fiber as a result of the smoothing of the sharp gradients. Nevertheless, it should be noted that the average fiber strain is generally well captured at the center of each fiber.

The average strain values given by the FE analyses in the loading direction are summarized in Table 2 for the composite, the matrix and the fibers. Comparison with the data obtained by DIC demonstrate the potential of this technique to accurately capture the average composite strain at high magnification using relatively large subset sizes and in the presence of marked strain non-homogeneities. DIC was not, however, capable of providing accurate values of the average fiber and matrix strains and tended to underestimate the matrix strain and to overestimate the fiber strain.

\section{Concluding remarks}

The application of DIC to analyzing the micromechanisms of deformation in fiber-reinforced composites was assessed from secondary electron micrographs obtained during mechanical tests within the scanning electron microscope. The study was carried out using micrographs with low magnification $(250 \times)$ and high magnification $(2000 \times$ and $6000 \times)$. At low magnification, the fibers themselves acted as the speckle pattern and the short elastic interactions between fibers as well as the sharp strain gradients at the fiber/matrix interfaces were completely smoothed out from the strain maps. However, the long range fluctuations in strain within the ROI due to the presence of matrix-rich and fiber-rich zones were captured at this magnification. It should be noted that this information is important so as to assess the degree of fiber clustering in the composite microstructure, that has been identified as one of the dominant factors controlling the onset of damage in composites [15]. In addition, the average composite strain measured by DIC was in good agreement with the values expected from the applied load and the transverse elastic modulus of the composite.

At higher magnifications, the speckle pattern was provided by a dispersion of submicron alumina particles on the specimen surface. DIC was able to accurately capture the displacement fields throughout the ROI and the strain fields obtained with DIC qualitatively reproduce the expected pattern: strains in the fibers were much smaller than those found in the matrix. In addition, maximum strain concentrations were found in the matrix between fibers aligned in the loading direction, the applied strain being mainly accommodated by the matrix ligaments between the fibers. Finally, the average composite strain at both magnifications was in very good agreement with the results of the accurate numerical simulations of the ROI by the finite element method. However, these simulations revealed that DIC was not able to accurately capture the average strain in each phase. It systematically underestimated the matrix strain while overestimating the fiber strain as a result of the smoothing of the sharp strain gradients at the fiber/matrix interface. It is obvious from these results that standard DIC can be used as a qualitative assessment tool at this magnification to determine the deformation and fracture mechanisms but reliable quantitative information can be only provided for the average composite properties in the ROI and not for the individual phases.

\section{Acknowledgements}

This investigation was supported by the Ministerio de Ciencia e Innovación de España through the Grant MAT 2009-14396, by the
Comunidad de Madrid through the program ESTRUMAT (S2009/ MAT-1585), by the research project DEFCOM (Era-Net MATERA, EU, 6th FP) and by the European Communitys Seventh Framework Programme FP7/2007-2013 under grant agreement 213371 (MAAXIMUS, http://www.maaximus.eu). In addition, the authors are indebted to Ms. B. Ferrari for her help in the preparation of the alumina nanoparticle suspension.

\section{References}

[1] Amsterdam E, De Hosson JTM, Onck PR. Failure mechanisms of closed-cell aluminium foam under monotonic and cyclic loading. Acta Mater $2006 ; 54: 4465-72$

[2] Anzelotti G, Nicoletto G, Riva E. Mesomechanic strain analysis of twill-weave composite lamina under unidirectional in-plane tension. Composites: Part A 2008;39:1294-301.

[3] Bornert M, Brémand F, Doumalìn P, Dupré J-C, Fazzini M, Grédiac M, et al Assessment of digital image correlation measurement errors: methodology and results. Exp Mech 2009;49:353-70.

[4] Doumalin P, Bornert M. Micromechanical applications of digital image correlation technique. In: Jacquot P, Fournier J, editors. Interferometry in speckle light: theory and application. Springer; 2000. p. 67-74.

[5] Ghadbeigi H, Pinna C, Celotto S, Yates JR. Local plastic strain evolution in a high strength dual-phase steel. Mater Sci Eng A 2010;527:5026-32.

[6] González C, Llorca J. Mechanical behavior of unidirectional fiber-reinforced polymers under transverse compression: microscopic mechanisms and modeling. Compos Sci Technol 2007;67:2795-806.

[7] Kang J, Ososkov Y, Embury JD, Wilkinson DS. Digital image correlation studies for microscopic strain distribution and damage in dual phase steels. Scripta Mater 2007:56:999-1002.

[8] Lagattu F, Bridier F, Villechaise P, Brillaud J. In-plane strain measurements on a microscopic scale by coupling digital image correlation and an in situ SEM technique. Mater Character 2006;56:10-8.

[9] Lomov S, Boisse P, Deluycker E, Morestin F, Vanclooster K, Vandepitte D, et al Full-field strain measurements in textile deformability studies. Composites: Part A 2008;39:1232-44

[10] Lomov SV, Ivanov DS, Verpoest I, Zako M, Kurashiki T, Nakai H, et al. Full field strain measurements for validation of meso-Fe analysis of textile composites. Composites: Part A 2008;39:1218-31.

[11] Molina-Aldaregúa JM, Rodríguez M, González C, LLorca J. An experimental and numerical study of the influence of local effects on the application of the fibre push-in test. Philos Mag 2011;91:1293-307.

[12] Peters WH, Ranson WF. Digital imaging techniques in experimental stress analysis. Opt Eng 1982;21:427-31.

[13] Réthoré J, Tinnes J-P, Roux S, Buffiêre J-Y, Hild F. Extended three-dimensional digital image correlation (X3D-DIC). Compt Rend Méc 2008;336:643-9.

[14] Ridruejo A, González C, Llorca J. Micromechanisms of deformation and fracture of polypropylene nonwoven fabrics. Int J Solids Struct 2011;48:153-62.

[15] Segurado J, González C, LLorca J. A numerical investigation of the effect of particle clustering on the mechanical properties of composites. Acta Mater 2003;51:2355-69.

[16] Segurado J, Llorca J. A computational micromechanics study of the effect of interface decohesion on the mechanical behavior of composites. Acta Mater 2005;53:4931-42.

[17] Sutton MA, Orteu JJ, Schreier HW. Image correlation for shape, motion and deformation measurements: basic concepts, theory and application. Springer; 2009.

[18] Chu TC, Ranson WF, Sutton MA, Peters WH. Applications of digital-imagecorrelation techniques to experimental mechanics. Exp Mech $1985 ; 25: 232-44$.

[19] Totry E, González C, LLorca J. Prediction of the failure locus of C/PEEK composites under transverse compression and longitudinal shear through computational micromechanics. Compos Sci Technol 2008;68:3128-36.

[20] Totry E, González C, LLorca J, Molina-Aldaregúia JM. Mechanisms of shear deformation in fiber-reinforced polymers: experiments and simulations. Int J Fract 2009;158:197-209.

[21] Totry E, Molina-Aldareguía IM González C Lorca J Effect of fiber, matrix and interface properties on the in-plane shear deformation of carbon-fiber reinforced composites. Compos Sci Technol 2010;70:970-80.

[22] Vendroux G, Knauss WG. Submicron deformation field measurements: Part 2 Improved digital image correlation. Exp Mech 1998;38:86-92.

[23] Wang Y, Cuitiño AM. Full-field measurements of heterogeneous deformation patterns on polymeric foams using digital image correlation. Int J Solids Struct 2002;39:3777-96.

[24] Yongqi S, Corletto C, Bradley WL, Tian J. Direct measurement of microscopic strain distribution near a crack tip. Exp Mech 1996;36:193-8. 